\title{
PRECONDITIONING IMPROVES CARDIOPLEGIA-RELATED CORONARY MICROVASCULAR SMOOTH MUSCLE HYPERCONTRACTILITY: ROLE OF $\mathrm{K}_{\text {ATP }}$ CHANNELS
}

Naruto Matsuda, $\mathrm{MD}, \mathrm{PhD}^{\mathrm{a}}$

Kathleen G. Morgan, $\mathrm{PhD}^{\mathrm{b}}$

Frank W. Sellke, MD
Objectives: The effect of preconditioning before hyperkalemic cardioplegia on the coronary smooth muscle remains to be elucidated. We tested the hypothesis that hypoxic preconditioning could protect coronary smooth muscle against subsequent hyperkalemic cardioplegia-induced coronary vasospasm and that this preconditioning effect could be mediated by $\mathbf{K}_{\mathrm{ATP}}$ channels. Methods: Rat coronary arterioles (endotheliumdenuded) were studied in a pressurized, no-flow, normothermic state. Simultaneous monitoring of luminal diameter and intracellular calcium concentration of vascular smooth muscle loaded with fura- 2 was made with microscopic image analysis. All vessels were subjected to $60 \mathrm{~min}$ utes of hypoxic hyperkalemic cardioplegia $\left(\mathrm{K}^{+}=\mathbf{2 5 . 0} \mathrm{mmol} / \mathrm{L}\right)$ and were then reperfused. Six groups were studied: (1) controls, no precardioplegic intervention; (2) preconditioning, achieved with 10 minutes of hypoxia $\left(\mathrm{Po}_{2}<30 \mathrm{~mm} \mathrm{Hg}\right)$ and 10 minutes of reoxygenation; (3) preconditioning plus glibenclamide $(10 \mu \mathrm{mol} / \mathrm{L})$, achieved with 10 minutes of preconditioning in the presence of $K_{\mathrm{ATP}}$ channel blocker glibenclamide; (4) pretreatment with $K_{\mathrm{ATP}}$ channel opener pinacidil (100 $\mu \mathrm{mol} / \mathrm{L})$; (5) pretreatment with pinacidil $(100 \mu \mathrm{mol} / \mathrm{L})$ plus glibenclamide (10 $\mu \mathrm{mol} / \mathrm{L})$; and (6) pretreatment with glibenclamide (10 $\mu \mathrm{mol} / \mathrm{L})$ alone. Results: Hypoxic preconditioning significantly $(P<.01)$ reduced hyperkalemic cardioplegia-induced intracellular calcium concentration accumulation and prevented the hypercontractility during and after hyperkalemic cardioplegia compared with control vessels. Pinacidil provided effective microvascular protection similar to hypoxic preconditioning. These vasoprotective effects of preconditioning were significantly antagonized in glibenclamide-treated vessels. Conclusions: Hypoxic preconditioning can prevent coronary microvascular hypercontractility during and after subsequent cardioplegia by a $K_{\mathrm{ATP}}$ channel mechanism that regulates intracellular calcium concentration of the vascular smooth muscle. (J Thorac Cardiovasc Surg 1999;118:438-45)
$\mathrm{H}^{\prime}$ yperkalemic cardioplegia $(\mathrm{K}-\mathrm{CP})$ is the most widely used technique for myocardial protection during cardiac operations. Although its effectiveness in protecting the cardiomyocyte against prolonged ischemia has been well established, it is frequently imperfect. A reason for suboptimal myocardial recovery may be related to reduced myocardial perfusion as the result of

From the Division of Cardiothoracic Surgery, Department of Surgery of Beth Israel Deaconess Medical Center, ${ }^{\mathrm{a}}$ and Harvard Medical School, Boston Biomedical Research Institute, ${ }^{\mathrm{b}}$ Boston, Mass.

Supported by grants (HL-46716 to F.W.S. and HL-31704 to K.G.M) from the National Institutes of Health.

Received for publication Nov 5, 1998; revisions requested Jan 26 , 1999; revisions received March 22, 1999; accepted for publication April 27, 1999. coronary vasospasm associated with $\mathrm{K}-\mathrm{CP} .{ }^{1-3}$ The state of coronary vasomotor tone may affect not only the distribution of cardioplegic solution during administration but also influence the coronary microcirculation in the postoperative setting.

Ischemic preconditioning has long been recognized to protect the heart from ischemia/reperfusion injury. 4

\footnotetext{
Address for reprints: Frank W. Sellke, MD, Division of Cardiothoracic Surgery, Beth Israel Deaconess Medical Center, East Campus, Dana 905, 330 Brookline Ave, Boston, MA 02215.
}

Copyright (C) 1999 by Mosby, Inc.

$0022-5223 / 99 \$ 8.00+0 \quad \mathbf{1 2 / 1 / 9 9 5 9 5}$ 
There is also clinical evidence that, during percutaneous transluminal coronary angioplasty or coronary bypass grafting, a brief period of ischemia similar to preconditioning may protect the heart from subsequent prolonged coronary occlusion and can reduce the incidence of myocardial infarction or severe myocardial dysfunction. ${ }^{5,6}$ Recently, some studies have demonstrated that the combination of ischemic preconditioning and cardioplegic arrest improved left ventricular pump function. ${ }^{7,8}$

We and others ${ }^{9-11}$ have recently reported beneficial effects of preconditioning on endothelial functional preservation. However, it remains unclear whether the protective effects of preconditioning also extends to coronary microvascular smooth muscle in the setting of $\mathrm{K}-\mathrm{CP}$. Similarly, although in a variety of mammalians including humans there has been accumulating evidence that an activation of adenosine triphosphate-sensitive $\mathrm{K}^{+}\left(\mathrm{K}_{\text {ATP }}\right)$ channels plays an important role in ischemic myocardial preconditioning, ${ }^{12-14}$ there is less consensus regarding the involvement of the $\mathrm{K}_{\text {ATP }}$ channel in the preconditioning phenomenon observed in coronary microvasculature.

To investigate the vasoprotective mechanisms of preconditioning adjuvant to $\mathrm{K}-\mathrm{CP}$, we established an in vitro isolated coronary microvessel model. Although this preparation presents interpretive limitations with regard to the in vivo coronary system, it does provide a suitable situation in which to perform real-time analyses of the microvascular tone and intracellular calcium dynamics in coronary arterioles during K-CP. Thus the aims of this study were to determine whether hypoxic preconditioning would limit the extent of cardioplegiarelated coronary vasospasm during and after subsequent $\mathrm{K}-\mathrm{CP}$ and, if so, whether activation of $\mathrm{K}_{\mathrm{ATP}}$ channels plays a role in the preserved microvascular function.

\section{Material and methods}

Isolated microvessel preparations. The methods for isolation of coronary microvessels were described previously. ${ }^{1}$ In brief, male Sprague-Dawley rats weighing between 350 and $400 \mathrm{~g}$ were anesthetized with chloroform. Sodium heparin (1000 IU/kg) was then administered intravenously. The heart was removed and placed into cold $\left(4^{\circ} \mathrm{C}\right)$ Krebs physiologic saline solution (Krebs-PSS), which consisted of the following ion concentrations (in millimoles per liter): $\mathrm{NaCl}$ 119.0, $\mathrm{NaHCO}_{3} 25.0, \mathrm{KCl} 4.6, \mathrm{KH}_{2} \mathrm{PO}_{4} 1.2, \mathrm{MgSO}_{4}$ $1.2, \mathrm{CaCl}_{2} 1.8$, and glucose 11.0. Coronary arterial microvessels $(80-130 \mu \mathrm{m}$ in internal diameter) were dissected from the left anterior descending artery-dependent subepicardial region in the left ventricle with a $\times 10$ to $\times 60$ dissecting microscope (Olympus Optical, Tokyo, Japan). During dissection, care was taken to remove as much of the surrounding myocardium as possible. The endothelium was removed selectively by advancement of a human hair (approximately $60 \mu \mathrm{m}$ in diameter) into the lumen to abrade the luminal surface, followed by the intraluminal injection of air bubbles. Microvessels were transferred to an experimental chamber in which both ends of the microvessel were cannulated with dual glass micropipettes (tip interior diameter, approximately $60 \mu \mathrm{m})$ and secured with 10-0 nylon monofilament suture (Ethicon, Inc, Somerville, NJ). The chamber was mounted on a transillumination system and oxygenated $(95 \%$ oxygen $/ 5 \%$ carbon dioxide); Krebs-PSS was continuously circulated through the tissue chamber. In all experiments, the absence of functional endothelium was tested by determining the responses to adenosine $5^{\prime}$-diphosphate $(100 \mu \mathrm{mol} / \mathrm{L})$ and sodium nitroprusside $(100 \mu \mathrm{mol} / \mathrm{L})$ in microvessels precontracted with U46619 $(0.1 \mu \mathrm{mol} / \mathrm{L})$. All vessels denuded of endothelium showed complete relaxation to sodium nitroprusside but failed to relax in response to adenosine diphosphate. All of the animals received humane care in compliance with the "Guide for the Care and Use of Laboratory Animals" published by the National Institute of Health (NIH Publication No. 85-23, revised 1985).

Internal luminal diameter measurements. The vessels were pressurized to $40 \mathrm{~mm} \mathrm{Hg}$ in a no-flow state with a burette manometer filled with Krebs-PSS. The internal luminal diameter was measured with a microscopic image system (Zeiss IM35 [Carl Zeiss, Inc, Jena, Germany] and Hitachi CCD TV camera, model KP-115 [Hitachi Ltd, Tokyo, Japan]). The calibration of the measurement was performed with an 80$\mu \mathrm{m}$ tungsten wire. The minimum resolution of the system was $1.5 \mu \mathrm{m}$.

Intracellular calcium concentration measurement. Intracellular calcium concentration of coronary microvascular smooth muscle was measured by the method described by Meininger and colleagues. ${ }^{15}$ Coronary microvessels in the tissue chamber were loaded with $5 \mu \mathrm{mol} / \mathrm{L}$ acetoxymethylester of fura-2 (fura-2/AM) in Krebs-PSS containing $0.05 \%$ dimethyl sulfoxide and $0.01 \%$ poloxamer (Pluronic F127; BASF Corp, Mt Olive, NJ). The loading time was 45 minutes followed by a 30 -minute wash period at $37^{\circ} \mathrm{C}$. The objective lens used was a Nikon Fluor $\times 40$ (numerical aperture, 0.8) (Nikon Corp, Tokyo, Japan). Optimal focus was adjusted to the middle of the vascular smooth muscle layer by a view of the vascular wall under a bright microscopic field. Excitation light at $350 \pm 5 \mathrm{~nm}$ and $390 \pm 6 \mathrm{~nm}$ was used. Emission at $510 \pm 24 \mathrm{~nm}$ was monitored with a photomultiplier tube (Hamamatsu R928; Hamamatsu Phonetics KK, Hamamatsu City, Japan) and digitized by a Data AcquisitionEZ A/D Converter (Data Translation, Inc, Marlboro, Mass). The digital signal of the 2 wavelengths was processed by a program written with the DTVee version 3.0 programming environment (Data Translation, Inc, Marlboro, Mass). Intracellular calcium concentration was estimated from the ratio $\left(\mathrm{R}_{350 / 390}\right)$ of measured fluorescence signals $(\mathrm{F})$ elicited at 2 wavelengths according to the equation:

$$
\mathrm{R}_{350 / 390}=\left(\mathrm{F} 350_{\mathrm{mv}}-\mathrm{F}_{550_{\mathrm{bg}}}\right) /\left(\mathrm{F} 390_{\mathrm{mv}}-\mathrm{F} 390_{\mathrm{bg}}\right)
$$




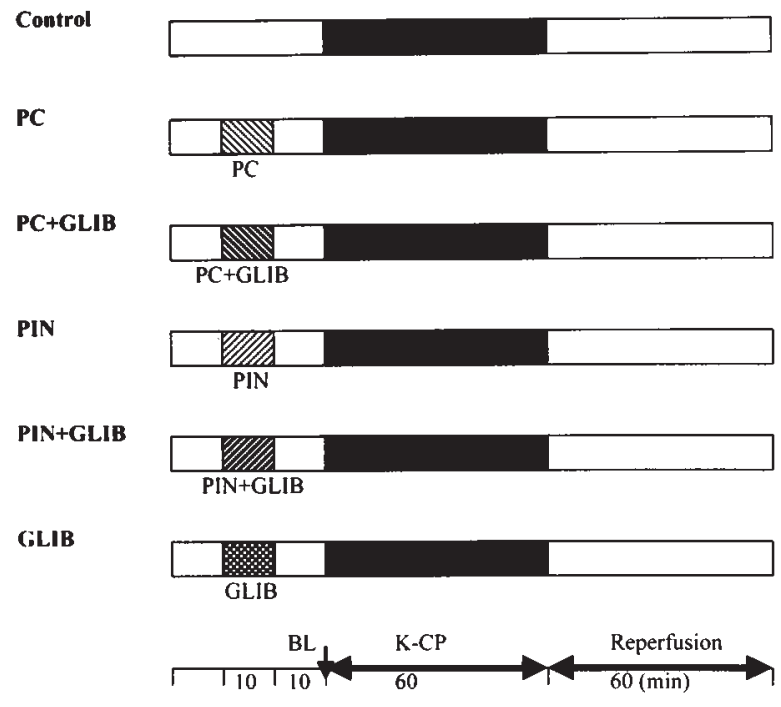

Fig 1. Schematic diagram of the experimental protocols. $P C$, Preconditioning; $P C+G L I B$, preconditioning in the presence of glibenclamide $(10 \mu \mathrm{mol} / \mathrm{L}) ; P I N$, pretreatment with pinacidil $(100 \mu \mathrm{mol} / \mathrm{L}) ; P I N+G L I B$, pretreatment with pinacidil $(100 \mu \mathrm{mol} / \mathrm{L})$ plus glibenclamide $(10 \mu \mathrm{mol} / \mathrm{L})$; $G L I B$, pretreatment with glibenclamide $(10 \mu \mathrm{mol} / \mathrm{L})$ alone; $B L$, baseline measurements.

where $\mathrm{F} 350_{\mathrm{mv}}$ and $\mathrm{F} 390_{\mathrm{mv}}$ are the total measured fluorescence of the microvessels at wavelengths of 350 and $390 \mathrm{~nm}$, and $\mathrm{F} 350_{\mathrm{bg}}$ and $\mathrm{F} 390_{\mathrm{bg}}$ are the background fluorescence signals at the respective wavelength. The background signals were measured on microvessel before loading fura-2/AM. Because there are a number of assumptions and concomitant uncertainties in absolute intracellular calcium concentration quantification with fura-2, on both practical and theoretic levels, ${ }^{16}$ the ratio $\left(\mathrm{R}_{350 / 390}\right)$ was used as an index of intracellular calcium concentration.

Experimental design. In an attempt to simulate the nonoxygenated crystalloid cardioplegia in the operating room, an in vitro hypoxic K-CP model was designed. Hypoxia (simulated ischemia) was used in the present study because ischemia (lack of blood flow) would not be an accurate term in regard to this continuously superfused microvascular model. After baseline measurement of intracellular calcium concentration and internal luminal diameter were taken, microvessels were subjected to 60 minutes of hypoxic K-CP of the following composition (in millimoles per liter): 121 $\mathrm{NaCl}, 25 \mathrm{KCl}, 12 \mathrm{NaHCO}_{3}, 1.2 \mathrm{CaCl}_{2}$, and 11.1 glucose and then reperfused with oxygenated Krebs-PSS for 60 minutes. Hypoxic condition was induced by switching bubbling gas from $95 \%$ oxygen $/ 5 \%$ carbon dioxide to $95 \%$ nitrogen $/ 5 \%$ carbon dioxide. The partial pressure of oxygen $\left(\mathrm{PO}_{2}\right)$ in the hypoxic $\mathrm{K}-\mathrm{CP}$ reached a stable level below $30 \mathrm{~mm} \mathrm{Hg}$ within 2 minutes after changing the bubbling gas. True anoxic condition was not achieved because a small amount of oxygen continuously diffused into the K-CP from the atmos- phere. The $\mathrm{pH}$ was $7.4 \pm 0.1$, and $\mathrm{PCO}_{2}$ was $35 \pm 5 \mathrm{~mm} \mathrm{Hg}$. The temperature was maintained at $37^{\circ} \mathrm{C}$ throughout the experiments.

Experimental protocols. The experimental protocols are depicted in Fig 1. Six groups were studied: (1) control, no pre-K-CP intervention; (2) preconditioning, achieved with 10 minutes of hypoxia in Krebs-PSS followed by 10 minutes of reoxygenation before the exposure of prolonged hypoxic $\mathrm{K}-\mathrm{CP}$; (3) preconditioning plus glibenclamide, achieved with preconditioning in the presence of $\mathrm{K}_{\mathrm{ATP}}$ channel blocker glibenclamide (10 $\mu \mathrm{mol} / \mathrm{L})$ before K-CP; (4) pinacidil, achieved by pretreatment with $\mathrm{K}_{\text {ATP }}$ channel opener pinacidil (100 $\mu \mathrm{mol} / \mathrm{L}$ ) before K-CP; (5) pinacidil plus glibenclamide, achieved by pretreatment with pinacidil $(100 \mu \mathrm{mol} / \mathrm{L})$ in the presence of glibenclamide (10 $\mu \mathrm{mol} / \mathrm{L})$ before $\mathrm{K}-\mathrm{CP}$; and (6) glibenclamide alone, achieved by pretreatment with glibenclamide $(10 \mu \mathrm{mol} / \mathrm{L})$ before K-CP.

Evaluation of microvascular function. After 60 minutes of reoxygenation, a cumulative concentration-response curve to a stable thromboxane $A_{2}$ analogue, U46619 $\left(10^{-9}-10^{-6} \mathrm{~mol} / \mathrm{L}\right)$ was constructed to evaluate the agonist-induced vascular contractility. Finally, papaverine $(100 \mu \mathrm{mol} / \mathrm{L})$ was applied in the tissue chamber to normalize the vascular diameter.

Drugs. Fura-2/AM and Pluronic F-127 were obtained from Molecular Probes (Eugene, Ore). Pinacidil was obtained from Research Biochemical International (Natick, Mass). Glibenclamide and U46619 were purchased from Sigma Chemical Company (St Louis, Mo). Papaverine was obtained from Eli Lilly \& Co (Indianapolis, Ind). Glibenclamide and pinacidil were dissolved in dimethyl sulfoxide initially and then added to the Krebs-PSS. The final concentration of dimethyl sulfoxide in the experimental solutions did not exceed $0.1 \%$.

Statistical analysis. The response of microvessels to each intervention was examined only once in each animal. Therefore each animal served as one sample. The changes in internal luminal diameter were expressed as percent contraction of the normalized diameter with application of papaverine. Results are expressed as mean \pm SD. The paired Student $t$ test was applied for within-group comparisons with baseline. Analysis of variance combined with a multiple-comparison Fisher's test was used to test the differences among groups with different interventions (StatView 4.0; Abacus Concepts Inc, Berkeley, Calif).

\section{Results}

Baseline characteristics before K-CP. Baseline measurements taken before the exposure to $\mathrm{K}-\mathrm{CP}$ are shown in Table I. Among the 6 groups, there were no significant differences in the baseline contraction and intracellular calcium concentration of microvascular smooth muscle, which suggests that each pretreatment protocol used in the present study did not cause significant changes in the steady-state microvascular tone.

Microvascular responses during K-CP. Fig 2 shows the representative time-course changes in vascu- 
Table I. Baseline values of internal luminal diameter and intracellular calcium concentration in coronary microvessels

\begin{tabular}{|c|c|c|c|}
\hline & $n$ & $\begin{array}{c}\text { Diameter } \\
(\%)\end{array}$ & $\begin{array}{c}\text { Intracellular } \\
\text { calcium concentration } \\
\left(R_{350 / 390}\right)\end{array}$ \\
\hline Control & 8 & $96.2 \pm 4.6$ & $0.42 \pm 0.04$ \\
\hline $\mathrm{PC}$ & 8 & $97.3 \pm 5.3$ & $0.41 \pm 0.04$ \\
\hline PC+GLIB & 8 & $95.7 \pm 5.5$ & $0.43 \pm 0.03$ \\
\hline PIN & 8 & $97.5 \pm 5.0$ & $0.42 \pm 0.04$ \\
\hline PIN+GLIB & 6 & $98.0 \pm 3.7$ & $0.43 \pm 0.05$ \\
\hline GLIB & 6 & $96.8 \pm 4.2$ & $0.42 \pm 0.04$ \\
\hline
\end{tabular}

Microvascular diameter was expressed as percentage of normalized diameter with papaverine $(100 \mu \mathrm{mol} / \mathrm{L})$. Intracellular calcium concentration was measured with fura-2 and expressed as the ratio of fluorescent signals at 350 and $390 \mathrm{~nm}\left(\mathrm{R}_{350 / 390}\right)$. All values are shown as mean $\pm \mathrm{SD}$. (See Fig 1 for group definitions.) There was no significant difference between groups (all $P>$.2).

lar contraction and intracellular calcium concentration during and after K-CP. In all vessels, vascular contraction increased gradually in a time-dependent manner, and these changes in vascular contraction were associated with changes in intracellular calcium concentration. After reperfusion, the vascular diameter and intracellular calcium concentration returned to baseline levels within approximately 15 minutes in all vessels.

To clarify the effects of each preconditioning protocol on the $\mathrm{K}-\mathrm{CP}$-induced vascular contraction, we focused on the responses at the end of 60 minutes of the $\mathrm{K}-\mathrm{CP}$ period (Fig 3). All vessels in the 6 groups exhibited significant increases both in vascular contraction and intracellular calcium concentration compared with respective baseline level $(P<.001)$. Hypoxic preconditioning caused a significant decrease both in the K$\mathrm{CP}-$ induced vascular contraction and intracellular calcium concentration elevation compared with control vessels (percent contraction, $25.2 \% \pm 5.8 \%$ vs $36.2 \% \pm$ 5.9\%; intracellular calcium concentration, $0.61 \pm 0.05$ vs $0.74 \pm 0.06$ in preconditioning vs control vessels, respectively; both $P<.01$ ). Furthermore, pinacidil pretreatment significantly reduced the $\mathrm{K}-\mathrm{CP}$-induced vascular contraction and intracellular calcium concentration elevation (percent contraction, $22.6 \% \pm 3.3 \%$; intracellular calcium concentration, $0.62 \pm 0.04$; both $P$ $<.01 \mathrm{vs}$ control). In marked contrast, coadministration of the $\mathrm{K}_{\text {ATP }}$ channel blocker glibenclamide completely reversed the effects of hypoxic preconditioning or pinacidil pretreatment. Pretreatment with glibenclamide alone did not exert significant effects on the K$\mathrm{CP}-$ induced vascular contraction and intracellular calcium concentration elevation compared with control group. These observations indicate that hypoxic pre-
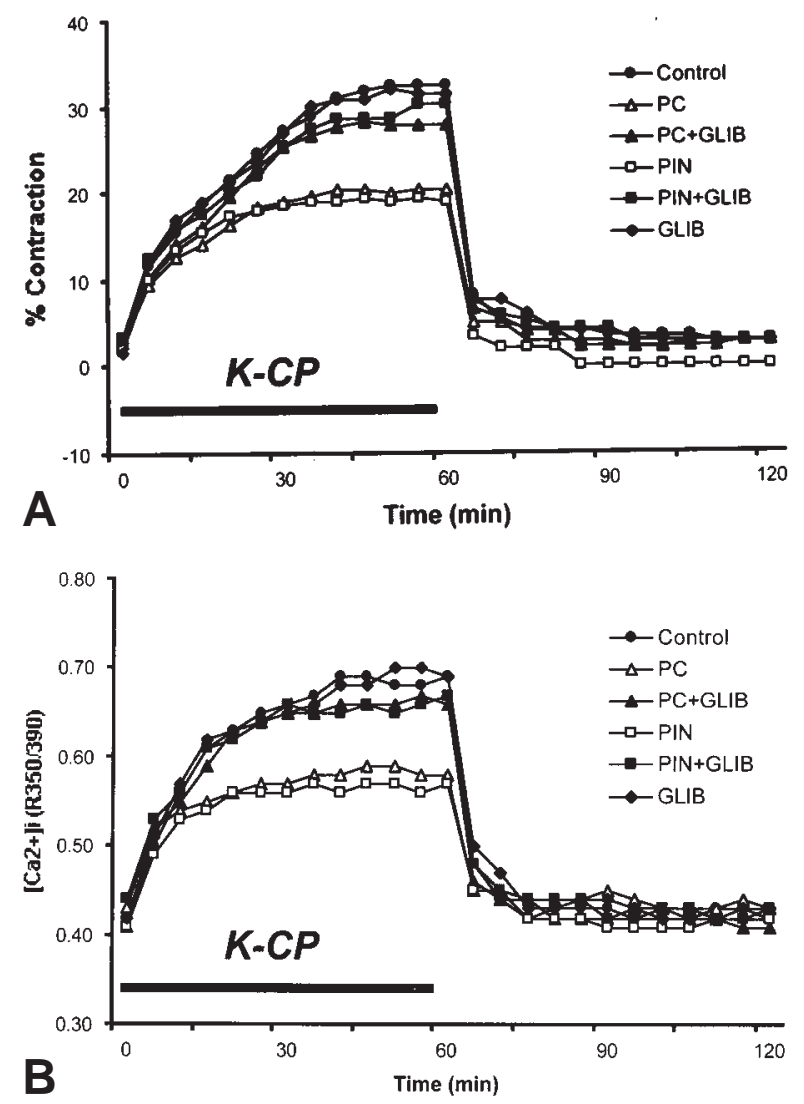

Fig 2. Representative time course profiles during and after K$\mathrm{CP}$ in (A) vascular contraction and (B) intracellular calcium concentration $([\mathrm{Ca} 2+] i)$ in vascular smooth muscle. Contractile responses were expressed as percent contraction of normalized diameter with papaverine $(100 \mu \mathrm{mol} / \mathrm{L})$. Intracellular calcium concentration was measured by fura-2 and expressed as the fluorescent ratio of F350/F390. (See Fig 1 for group definitions.)

conditioning can attenuate $\mathrm{K}-\mathrm{CP}$-induced vascular contraction through a $\mathrm{K}_{\mathrm{ATP}}$ channel mechanism, which reduces intracellular calcium concentration accumulation in coronary smooth muscle.

Microvascular function after K-CP/reperfusion. The data presented in Fig 4 illustrate the agonistinduced contractile responses to U46619 at the end of 60 minutes of reperfusion. A separate group $(n=8)$ of rats were used to measure normal contractility (baseline response) of vessels not exposed to K-CP. U46619evoked vessel contractility was significantly enhanced in control and the 3 glibenclamide-administered groups compared with the baseline response (all $P<.05$ ). On hypoxic preconditioned or pinacidil-pretreated vessels, vascular contractility elicited by U46619 was entirely preserved in a baseline response level. The results sug- 

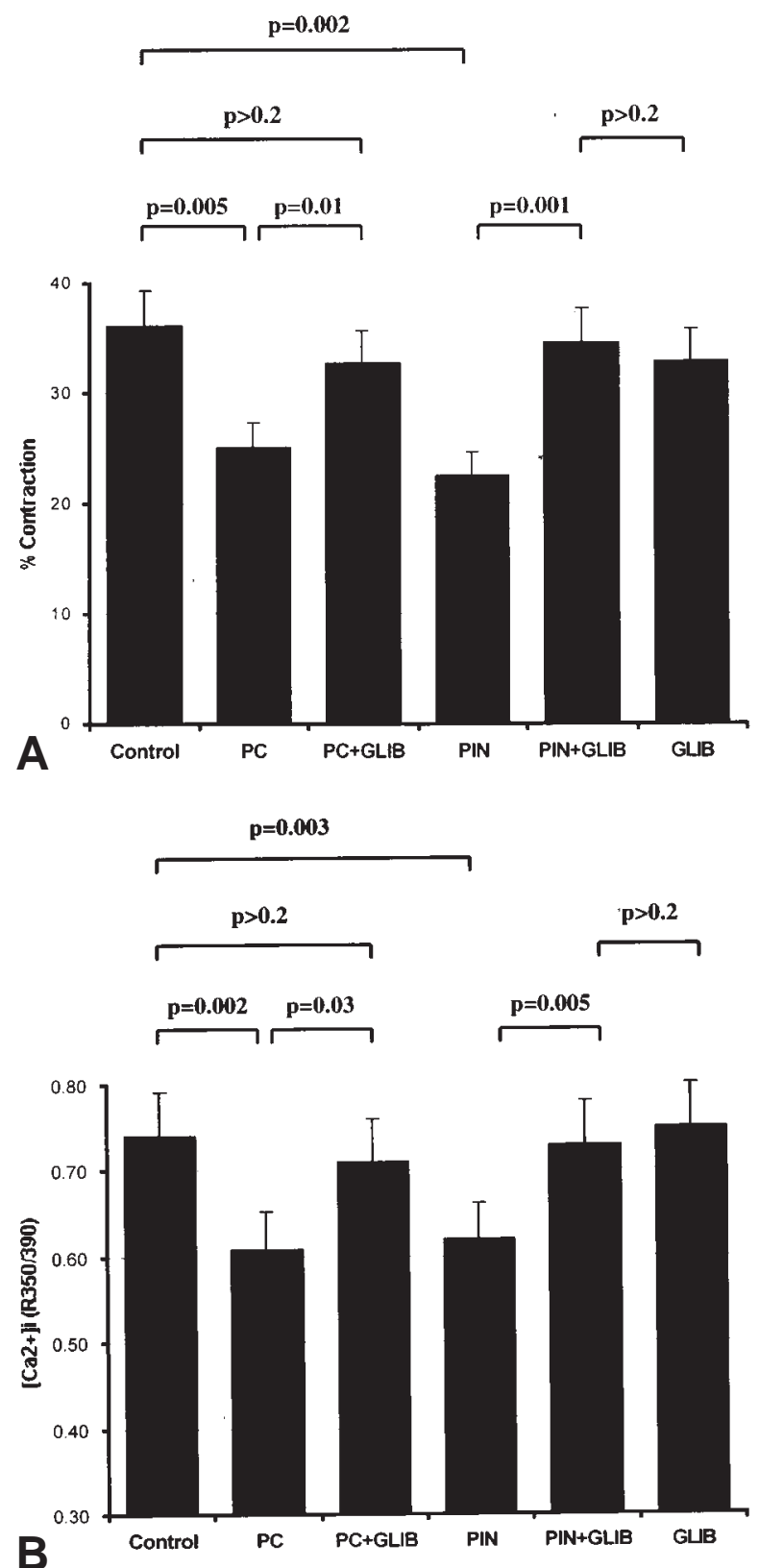

Fig 3. Comparisons in (A) vascular contraction and (B) intracellular calcium concentration $([\mathrm{Ca} 2+] i)$ in vascular smooth muscle at the end of 60 minutes of K-CP. Contractile responses were expressed as percent contraction of normalized diameter with papaverine $(100 \mu \mathrm{mol} / \mathrm{L})$. Intracellular calcium concentration was measured by fura- 2 and expressed as the fluorescent ratio of F350/F390. All values are shown as means \pm SD. (See Fig 1 for group definitions.) Statistically significant $P$ values are given.

gest that hypoxic preconditioning or pinacidil pretreatment preserved physiologic level of agonist-induced vascular contractility after $\mathrm{K}-\mathrm{CP} /$ reperfusion.

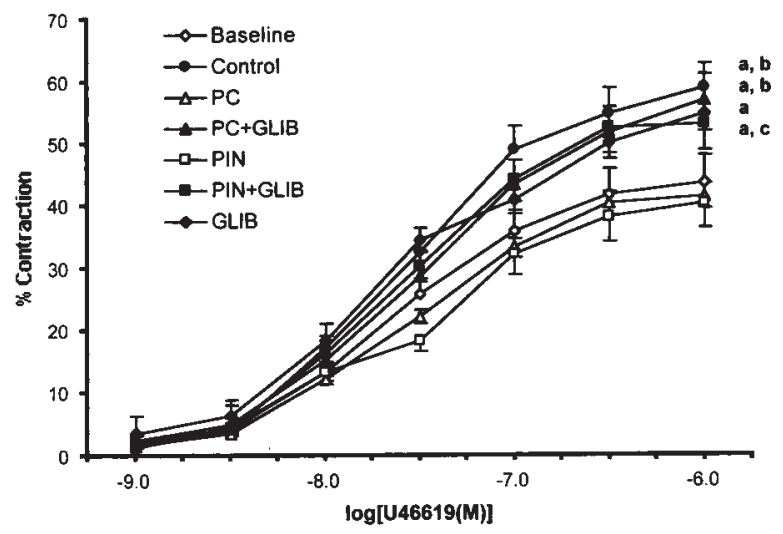

Fig 4. Concentration-response curve to receptor-mediated vasoconstrictor U46619 after K-CP and reperfusion. Contractile responses were expressed as percent contraction of normalized diameter with papaverine $(100 \mu \mathrm{mol} / \mathrm{L})$. All values are shown as means \pm SD. (See Fig 1 for group definitions.) Baseline, Baseline response, vessels not exposed to hypoxic K-CP. ${ }^{\mathrm{a}} P<.05$ vs Baseline; ${ }^{\mathrm{b}} P<.05$ vs $\mathrm{PC} ;{ }^{\mathrm{c}} P=.02$ vs PIN.

\section{Discussion}

The unique findings of this study were 2-fold. First, hypoxic preconditioning reduced the intracellular calcium concentration accumulation in coronary vascular smooth muscle and attenuated the vascular contraction during subsequent hypoxic $\mathrm{K}-\mathrm{CP}$ and prevented microvascular smooth muscle hypercontractility after $\mathrm{K}-\mathrm{CP} /$ reperfusion. Second, this protective effect of hypoxic preconditioning can be mimicked by pharmacologic activation of $\mathrm{K}_{\text {ATP }}$ channels with pinacidil.

Effect of hypoxic preconditioning on $\mathrm{K}-\mathrm{CP}-$ induced vascular contraction. Ischemic preconditioning refers to the observation that brief periods of ischemia and reperfusion protect the myocardium from injury during subsequent more severe ischemic insults. ${ }^{4}$ This phenomenon has been described in a number of species. It has been known that preconditioning stimuli are species specific. In this study, we used rat heart because preconditioning has been shown to confer myocardial functional protection in this species. ${ }^{17,18}$

Several recent studies have demonstrated that the beneficial effect of preconditioning is not limited to the cardiomyocytes but can be observed in endothelial cells in various experimental models, including dog resistance coronary arteries in vivo ${ }^{10}$ and conduit coronary arteries of the rat in vitro. ${ }^{11}$ We have already reported that temporary ischemia/reperfusion before prolonged ischemia could preserve endothelial function in pig coronary microvessels ${ }^{9}$; however, whether the microvascular smooth muscle of the coronaries is included in the preconditioning phenomenon remains 
unresolved. Such distinction between endothelium and vascular smooth muscle would be essential to understand the precise nature of vascular preconditioning phenomenon. Therefore we used an endotheliumdenuded rat coronary microvessel model to investigate vascular smooth muscle response to hypoxic preconditioning in conditions of simulated hyperkalemic cardioplegic arrest in cardiac operations. Our results demonstrated that preconditioning was able to attenuate the coronary smooth muscle hypercontractility associated with hypoxic K-CP.

In the present study, we examined intracellular calcium concentration in vascular smooth muscle to investigate the cellular and molecular mechanisms of the vascular preconditioning phenomenon against $\mathrm{K}-\mathrm{CP}-$ induced vascular contraction. Our experiments with hypoxic preconditioning clearly show that attenuation of the $\mathrm{K}-\mathrm{CP}-$ induced contraction is associated with a decrease in calcium concentration accumulation in vascular smooth muscle. This suggests that hypoxic preconditioning profoundly influences the intracellular calcium concentration regulation systems in vascular smooth muscle.

The basic mechanism responsible for the intracellular calcium concentration accumulation in vascular smooth muscle during $\mathrm{K}-\mathrm{CP}$ is related to membrane depolarization on the basis of Nernst's equation of membrane potential. ${ }^{19}$ Membrane depolarization promotes calcium concentration influx through voltage-dependent calcium concentration channels. Calcium concentration influx can also induce release of calcium concentration from intracellular calcium concentration stores. ${ }^{20}$ In addition, during surgical cardioplegic arrest, especially with nonoxygenated crystalloid K-CP, coronary microvessels are exposed to conditions of hypoxia. This would be associated with a lower production of adenosine triphosphate compared with a normoxic state. To date, intracellular calcium concentration elevation with hypoxia has been reported in many smooth muscles. In previous studies, it was suggested that calcium concentration release from the sarcoplasmic reticulum may contribute to hypoxic pulmonary vasoconstriction. ${ }^{21,22}$ Thus it is tempting to speculate that hypoxic preconditioning stimuli sensitizes calcium concentration efflux mechanisms or minimizes calcium concentration influx into vascular smooth muscle and thereby reduces vascular contraction during and after hypoxic K-CP. However, the exact mechanism by which preconditioning improves intracellular calcium concentration homeostasis against $\mathrm{K}-\mathrm{CP}$ is not clear at this time and requires further investigation.

Possible mechanism of vascular preconditioning before K-CP. In the experiments presented here, pre- treatment with $\mathrm{K}_{\text {ATP }}$ channel opener pinacidil significantly reduced the $\mathrm{K}-\mathrm{CP}-$ induced calcium concentration accumulation in smooth muscle and that was associated with a significant attenuation of $\mathrm{K}-\mathrm{CP}-$ induced vascular contraction. One possible explanation for this finding is that hyperpolarization of the smooth muscle cell membrane as the result of the opening of $\mathrm{K}_{\mathrm{ATP}}$ channels can be expected to reduce the inward calcium concentration current through voltage-sensitive calcium concentration channels ${ }^{23}$ and, consequently, to maintain the intracellular calcium concentration level during the subsequent period of sustained hypoxic KCP.

Another important finding presented in this study is that agonist (U46619)-induced microvascular contractile responses after K-CP were significantly enhanced in nonpreconditioned control vessels, whereas normal contractility was preserved in hypoxic preconditioned or pinacidil-pretreated vessels. The exact mechanisms underlying post-K-CP vascular hypercontractility are not clear; however, it may be related to the reduced calcium concentration accumulation in the vascular smooth muscle during K-CP. As shown in the nonpreconditioned control vessels, elevated intracellular calcium concentration during $\mathrm{K}-\mathrm{CP}$ may subsequently stimulate activation of calcium concentration-dependent intracellular signal pathways and may increase the calcium concentration sensitivity of the contractile apparatus after K-CP. Previous studies have demonstrated that the agonist-induced vascular tone is regulated by myosin light chain kinase, whose activity is governed by a calcium concentration calmodulin-mediated phosphorylation. ${ }^{24}$ There is some evidence that intracellular calcium concentration may also directly activate the calcium concentration-dependent isoforms of protein kinase $\mathrm{C}$ (conventional protein kinase $\mathrm{C}$ ) and lead directly or indirectly to the phosphorylation of an entirely different subset of cellular proteins including caldesmon, a number of intermediate filament proteins (desmin, synemin), and a few cytosolic proteins. ${ }^{25}$ Therefore it is reasonable to speculate that the intracellular calcium concentration accumulation in microvascular smooth muscle during K-CP becomes a strong trigger for post-K-CP coronary hypercontractility.

Clinical implication and limitation of this study. Myocardial preconditioning has been suggested to have adjuvant cardioprotective effects with cardioplegic arrest and reperfusion., ${ }^{7,8}$ In addition, the approach of pharmacologic preconditioning with $\mathrm{K}_{\mathrm{ATP}}$ channel openers may be of special interest because these drugs are both clinically accessible and acceptable. Several studies have indeed documented an improved recovery of myocardial function treated with 
$\mathrm{K}_{\text {ATP }}$ channel openers given before or during a sustained episode of global ischemia with or without cardioplegia. ${ }^{26-28}$ In the present study, we showed that pharmacologic activation of $\mathrm{K}_{\mathrm{ATP}}$ channels could attenuate vasoconstriction during and after $\mathrm{K}-\mathrm{CP}$, which may improve not only the distribution of the cardioplegic solution during administration but also the coronary microcirculation after reperfusion. Thus $\mathrm{K}_{\mathrm{ATP}}$ channel openers may have novel clinical applications in achieving optimal myocardial protection for cardiac surgical procedures.

The present study was designed to provide direct evidence about beneficial effects of ischemic preconditioning on coronary smooth muscle function. Although the isolated coronary microvessel model used in the present study has some advantages over an in vivo preparation, we acknowledge some methodologic limitations inherent in the experimental design, including the hypoxic preconditioning as a simulation of ischemic preconditioning. Furthermore, the results of this study may be limited by the fact that they were obtained under conditions of nonoxygenated crystalloid cardioplegia in normoxia. In light of these limitations, the next logical step is to see whether the same patterns of vascular responsiveness are observed under cold and/or oxygenated cardioplegic conditions.

\section{Conclusions}

Our experiments performed in an endotheliumdenuded isolated rat coronary microvessel model demonstrated that preconditioning provides protective effects on coronary microvascular function during and after subsequent hypoxic K-CP by a mechanism that regulates intracellular calcium concentration of vascular smooth muscle with activation of $\mathrm{K}_{\mathrm{ATP}}$ channels. The present study may suggest an important new avenue to minimize the development of coronary vasospasm, particularly in the setting of hyperkalemic cardioplegic arrest under cardiac operations.

\section{REFERENCES}

1. Sellke FW, Shafique T, Schoen EJ, Weintraub RM. Impaired endothelium-dependent coronary microvascular relaxation following cold potassium cardioplegia and reperfusion. J Thorac Cardiovasc Surg 1993;105:52-8.

2. Sellke FW, Shafique T, Ely DL, Weintraub RM. Coronary endothelial injury following cardiopulmonary bypass and ischemic cardioplegia is mediated by oxygen-derived free radicals. Circulation 1993;88:395-400.

3. Keller MW, Geddes L, Spotnitz W, Kaul S, Duling BR. Microcirculatory dysfunction following perfusion with hyperkalemic, hypothermic cardioplegic solutions and blood reperfusion: effects of adenosine. Circulation 1991;84:2485-94.
4. Murry, CE, Jennings RB, Reimer KA. Preconditioning with ischemia: a delay of lethal cell injury in ischemic myocardium. Circulation 1986;74:1124-36.

5. Deutsch E, Berger M, Kussmaul WG, Hirshfeld JW Jr, Herrmann HC, Laskey WK. Adaptation to ischemia during percutaneous transluminal coronary angioplasty: clinical, hemodynamic, and metabolic features. Circulation 1990;82:2044-51.

6. Alkhulaifi AM, Yellon DM, Pugsley WB. Preconditioning the human heart during aorto-coronary bypass surgery. Eur J Cardiothorac Surg 1994;8:270-6.

7. Illes RW, Wright JK, Inners-McBride K, Yang CJ, Tristan A. Ischemic preconditioning improves preservation with crystalloid cardioplegia. Ann Thorac Surg 1994;58:1481-5.

8. Ogino H, Smolenski RT, Zych M, Seymour AML, Yacoub MH. Influence of preconditioning on rat heart subjected to prolonged cardioplegic arrest. Ann Thorac Surg 1996;62:469-74.

9. Tofukuji M, Metais C, Li J, Hariawala MD, Franklin A, Vassileva $\mathrm{C}$, et al. Effects of ischemic preconditioning on myocardial perfusion, function, and microvascular regulation. Circulation 1998; 98(suppl):II197-204.

10. DeFily DV, Chilian WM. Preconditioning protects coronary arteriolar endothelium from ischemia-reperfusion injury. Am J Physiol 1993;265:H700-6.

11. Richard V, Kaeffer N, Tron C, Thuillez C. Ischemic preconditioning protects against coronary endothelial dysfunction induced by ischemia and reperfusion. Circulation 1994;89:1254-61.

12. Gross GJ, Auchampach JA. Blockade of ATP-sensitive potassium channels prevents myocardial preconditioning in dogs. Circ Res 1992;70:223-33.

13. Schulz R, Rose J, Heusch G. Involvement of activation of ATPdependent potassium channels in ischemic preconditioning in swine. Am J Physiol 1994;267:H1341-52.

14. Speechly-Dick ME, Grover GJ, Yellon DM. Does ischemic preconditioning in the human involve protein kinase $\mathrm{C}$ and the ATPdependent $\mathrm{K}^{+}$channel? Studies of contractile function after simulated ischemia in an atrial in vitro model. Circ Res 1995;77: 1030-5.

15. Meininger GA, Zawieja DC, Falcone JC, Hill MA, Davey JP. Calcium measurement in isolated arterioles during myogenic and agonist stimulation. Am J Physiol 1991;261:H950-9.

16. Grynkiewicz G, Poenie M, Tsien RY. A new generation of $\mathrm{Ca}^{2+}$ indicators with greatly improved fluorescent properties. J Biol Chem 1985;260:3440-50.

17. Asimakis GK, Inners-McBride K, Medellin G, Conti VR. Ischemic preconditioning attenuates acidosis and postischemic dysfunction in isolated rat heart. Am J Physiol 1992;263:H887-94.

18. Liu Y, Downey JM. Ischemic preconditioning protects against infarction in rat heart. Am J Physiol 1992;263:H1107-12.

19. Powell T, Tatham PER, Twist VW. Cytosolic free calcium measured by Quin2 fluorescence in isolated ventricular myocytes at rest and during potassium-depolarization. Biochem Biophys Res Commun 1984;122:1012-20.

20. Fabiato A. Simulated calcium current can both cause calcium loading in and trigger the calcium release from the sarcoplasmic reticulum of skinned cardiac cell. J Gen Physiol 1985;85:291320.

21. Liu X, Engelman RM, Wei Z, Maulik N, Rousou JA, Flack JE III, et al. Postischemic deterioration of sarcoplasmic reticulum: warm vs cold blood cardioplegia. Ann Thorac Surg 1993;56:1154-9.

22. Gelband $\mathrm{CH}$, Gelband H. $\mathrm{Ca}^{2+}$ release from intracellular stores is 
an initial step in hypoxic pulmonary vasoconstriction of rat pulmonary artery resistance vessels. Circulation 1997;96:3647-54.

23. Quast U. Do the $\mathrm{K}^{+}$channel openers relax smooth muscle by opening $\mathrm{K}^{+}$channels? Trends Pharmacol Sci 1993;14:332-7.

24. Sasaguri T, Itoh T, Hirata M, Kitamura K, Kuriyama H. Regulation of coronary artery tone in relation to the activation of signal transducers that regulate calcium homeostasis. J Am Coll Cardiol 1987;9:1167-78.

25. Rasmussen H, Takuwa Y, Park S. Protein kinase C in the regulation of smooth muscle contraction. FASEB J 1987;1:17785 .
26. Qiu Y, Galinanes M, Hearse DJ. Protective effect of nicorandil as an additive to the solution for continuous warm cardioplegia. J Thorac Cardiovasc Surg 1995;110:1063-72.

27. Maskal SL, Cohen NM, Hsia PW, Wechsler AS, Damiano RJ. Hyperpolarized cardiac arrest with a potassium-channel opener, aprikalim. J Thorac Cardiovasc Surg 1995;110:1083-95.

28. Guo AC, Diacono J, Feuvray D. Comparison of effects of aprikalim and of hypoxic and ischemic preconditioning on extracellular potassium accumulation, metabolism and functional recovery of the globally ischemic rat heart. Cardiovasc Res 1994;28:864-71.

\section{Bound volumes available to subscribers}

Bound volumes of The Journal of Thoracic and Cardiovascular Surgery are available to subscribers (only) for the 1999 issues from the Publisher, at a cost of $\$ 134.00$ for domestic, $\$ 165.85$ for Canadian, and $\$ 155.00$ for international subscribers for Vol 117 (January-June) and Vol 118 (July-December). Shipping charges are included. Each bound volume contains a subject and author index and all advertising is removed. Copies are shipped within 60 days after publication of the last issue of the volume. The binding is durable buckram with the Journal name, volume number, and year stamped in gold on the spine. Payment must accompany all orders. Contact Mosby, Inc, Subscription Services, 11830 Westline Industrial Drive, St Louis, MO 63146-3318, USA; phone 800-453-4351 or 314-453-4351.

Subscriptions must be in force to qualify. Bound volumes are not available in place of a regular Journal subscription. 